\title{
Comparison Of Reliability Prediction Methods Using Life Cycle Cost Analysis
}

\author{
Adithya Thaduri, Lulea University of Technology \\ A K Verma, $\mathrm{PhD}$, Stord/Haugesund University College \\ Uday Kumar, PhD, Lulea University of Technology
}

Keywords: 217+, BJT Transistor, Instrumentation Amplifier, Life cycle cost, Physics of Failure, Reliability prediction

\section{SUMMARY AND CONCLUSION}

In this paper, it was discussed on the several reliability prediction models for electronic components and comparison of these methods was also illustrated. A combined methodology for comparing the cost incurring for prediction was designed and implemented with an instrumentation amplifier and a BJT transistor. By using the physics of failure approach, the dominant stress parameters were selected on basis of research study and were subjected to both instrumentation amplifier and BJT transistor. The procedure was implemented using the methodology specified in this paper and modeled the performance parameters accordingly. From the prescribed failure criteria, mean time to failure was calculated for both the components. Similarly, using 217 plus reliability prediction book, MTTF was also calculated and compared with the prediction using physics of failure. Then, the costing implications of both the components were discussed and compared them. From the results, it was concluded that for critical components like instrumentation amplifier though the initial cost of physics of failure prediction is too high, the total cost incurred including the penalty costs were lower than that of traditional reliability prediction method. But for non-critical components like BJT transistor, the total cost of physics of failure approach was too higher than traditional approach and hence traditional approach was much efficient. Several other factors were also compared for both reliability prediction methods.

\section{INTRODUCTION}

The important considerations for the customer to select an item depend on the reliability, cost, availability and maintainability. To deal with the perfect repair/replacement costs, selection of the time to repair or time to replace was properly calculated or else inaccuracy in reliability prediction of this values leads to increase in the excessive costs even the actual component has higher reliability. Hence, reliability prediction of these components along with life cycle costs was needed to be considered for effective working of the system. Accordingly, there would not be same replacement times for all the components with variable reliability and costs due to the variability in criticality of the each component. This paper concentrates on this issue on which reliability prediction method was to be selected for calculation of replacement human factors, time and reputation costs.

The efficient reliability prediction is needed before the installation of the components and appropriate changes can be made at the design stages. Conventional reliability prediction methods such as Mil-Hdbk 217F, Telcordia, Bell Core, PRISM etc implements constant failure methods and believed to be true in the era of 1980s and 1990s [3]. But due to the advancements in the technology, these methods are no longer adequate to define the characteristics as there are so much variability in the design and fabrication of devices. Especially, electronics spreads out rapid developments in all the aspects and for control aspects, miniature and cost effectiveness. Some of the devices are used in safety, security and military areas where the availability is the major concern and incorrect operation leads to the unsafe shutdown. Moreover reliability aspects and prediction is critical for these components and this paper provides advanced physics of failure methodology for finding failure characteristics and reliability indices. The following Table 1 demonstrates various traditional prediction methods the differences between the values of time to failures of DC-DC converter constraints the ambiguity and risk in selecting appropriate figure [1].

\section{Table 1: Comparisons of different reliability prediction} models [1]

\begin{tabular}{|c|c|c|c|c|c|c|c|}
\hline \multirow[t]{3}{*}{ Reliability Prediction Model $^{1}$} & \multirow[t]{3}{*}{ Company } & \multicolumn{4}{|c|}{1 Watt DC-DC Converter ${ }^{2}$} & \multirow{2}{*}{\multicolumn{2}{|c|}{$\begin{array}{c}100 \mathrm{~W} \\
\text { AC-DC PSU } \\
40^{\circ} \mathrm{C}\end{array}$}} \\
\hline & & \multicolumn{2}{|c|}{$25^{\circ} \mathrm{C}$} & \multicolumn{2}{|c|}{$85^{\circ} \mathrm{C}$} & & \\
\hline & & Hours & Years $^{4}$ & Hours & Years $^{4}$ & Hours & Years $^{4}$ \\
\hline MIL-HDBK-217F (EXAR 7.0) & A & $31,596,574$ & 3606.9 & & & 686,771 & 78.4 \\
\hline MIL-HDBK-217F Notice 2 & B & 832,000 & 95.0 & 86,000 & 9.8 & & \\
\hline MIL-HDBK-217F Notice 1 & $\mathrm{C}$ & 156,000 & 17.8 & 124,000 & 14.2 & & \\
\hline Telcordia SR332 Parts count & $\mathrm{D}$ & $89,380,000$ & 10203.2 & $29,260,000$ & 3340.2 & & \\
\hline Telcordia SR332 Parts stress & D & $104,200,000$ & 11895.0 & $57,160,000$ & 6525.1 & & \\
\hline Siemens SN29500 (IEC61709) & A & \begin{tabular}{|l|l|}
$80,978,217$ \\
\end{tabular} & 9244.1 & & & $1,554,055$ & 177.4 \\
\hline HRD5 Parts stress & $\mathrm{B}$ & $2,465,000$ & 281.4 & 849,000 & 96.9 & & \\
\hline HRD4 Parts count & $\mathrm{B}$ & $1,132,000$ & 129.2 & $1,132,000$ & 129.2 & & \\
\hline MIL-HDBK-217F (EXAR 7.0) & A & $31,596,574$ & 3606.9 & & & 686,771 & 78.4 \\
\hline Telcordia SR332 Parts count & E & & & & & $1,418,000$ & 162.0 \\
\hline
\end{tabular}

Physics of failure prediction methodology lay emphasis on the root cause of failure following fundamentals of physics of materials also considered as white box testing. Electronic devices were fabricated of different materials like Silicon, 
Germanium, Copper, Aluminum etc., with predefined technology and processes like deposition, etching and masking under controlled environment [5]. Most of failures happened due to changes in thermal activation, changes in defects of molecules, activation energy in the materials and correspondingly there was degradation in the technology and performance factors. Depends on the failure characteristics, several failure mechanisms were modeled and categorized according to the cause, material, and failure point location etc., produced in the literature. This method requires sophisticated tools for failure analysis and advanced tools for analyzing the simulated data. Still, this methodology also has challenges like insufficient data from the manufacturer, needs expert judgment and also time taking process.

\section{Figure 1: Short idea of Modified approach}

On the other hand, statistical methods were widely available in order to find out the reliability indices from the test data. This method was also considered as black box testing which concentrate on available data and proper model was selected depends on the application. There were possibilities to analyze the data and generated model to extract enormous amount of information to characterize the performance parameters. Some of them include design of experiments, accelerated testing, regression analysis, etc. Even, there were several tools available for model selection, mathematical formulation and model analysis. This methodology has some advantages like time consuming, no need for manufacturer data and parameter analysis.

Inclusion of multidisciplinary science and engineering approaches was very effective in solving of real life problems and our modified approach was combination of both physics of failure (deterministic) and statistical (probabilistic) approaches in Figure 1. This advancement methodology first starts with the proper understanding of basic failure physics of the component and process the physics of failure methodology. This knowledge was fed to the statistical approach to further refining of data for accurate models. Finally, we get three faces of models; history and literature, white box and black box models and these were sent to decision support system. Simultaneously, the cost of each prediction method was also compared for the decision on which method needed to be implemented on the criticality of the component. Other hidden costs include the amount of time that was spent on the entire processing of the prediction as it also takes care of the entire decision strategy. These details were also incorporated in this paper. The next section provides the entire block diagram for the reliability prediction with life cycle costs of each stage.

\section{METHODOLOGY}

The block diagram shown in Figure 2 describes the flow of prediction mechanism adopted in this paper.

\subsection{Component Description}

The first step in this methodology was to describe the component with all the necessary and essential information for analyzing the failure and calculating the reliability indices. The sources required for the information on the component were: materials, processes, layout diagrams, technology, architecture, design, criticality, cost, datasheet, manuals, field data and any similar item analysis that was analyzed earlier.

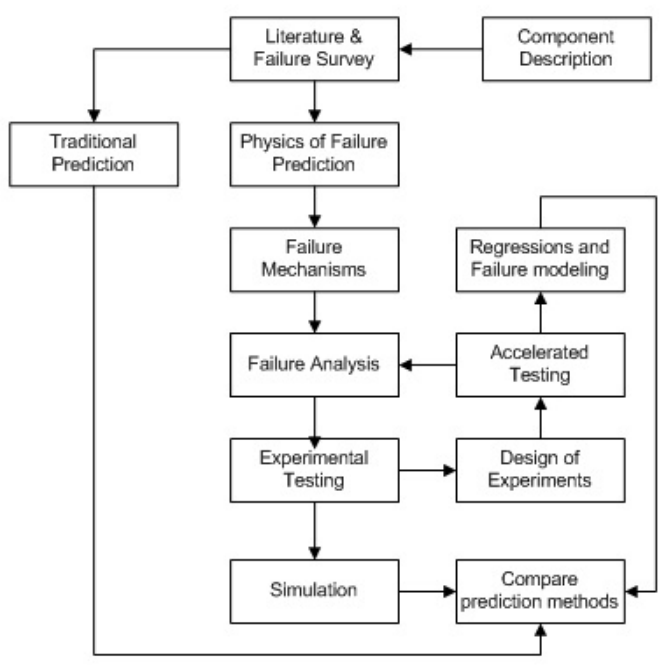

Figure 2: Reliability prediction methodology for comparisons

\subsection{Literature Survey and Failure Survey}

To study the failure behavior of the component, the literature survey was required to understand the behavior of several factors that affecting the performance. The information essential for this study were: expert reviews, stress factors, failure criteria, failure mechanisms, failure modes, failure analysis, degradation analysis and other factors.

\subsubsection{Traditional Reliability Methodology}

Based on the operational stresses and field environment, reliability indices were calculated using MilHdbk 217F and other traditional methods.

\subsubsection{Physics of Failure Methodology}

The advanced methodology, physics of failure lay emphasis on the root cause of failure inherently depends on the operational stress factors, environment and physical characteristics of the device [5]. From the information on component description and literature survey, appropriate failure phenomenon and failure criteria were selected. 


\subsection{Failure Mechanisms}

There was several failure mechanisms reported in the literature characterized on operational environment, stress parameters, level of approach, technology etc. Some of list of the failure mechanisms that was not limited to electromigration, hot carrier defects, time dependent dielectric breakdown, negative bias temperature instability, corrosion, fatigue, solder reliability, stress migration, soft errors (radiation effects) etc $[5,6]$. There were several failure time to failure models associated with each mechanism and appropriate model was picked for the application. According to the selected component, the appropriate failure mechanism or degradation mechanisms were studied.

\subsection{Failure Analysis}

From the literature, an appropriate failure analysis was selected to examine and illustrate the failure of the component and root cause of failure by electrical characterization or by using non-destructive testing by making use of sophisticated instruments like scanning electron microscope, infrared spectroscopy, thermal analysis etc [9]. For some of the components where there was no information on the failure mechanism, this step was need to be implemented beforehand to acquire information on failure characteristics.

\subsubsection{Experimental Planning}

From the acquired data, the next process of experimentation was planned for testing and reliability prediction. The desired circuitry was designed and fabricated using printed circuit board. This step also includes the number of samples, stress parameters and experimental setup for further testing of the component.

\subsubsection{Simulations}

There are several tools were available to carry out simulations depends on finite element analysis, circuit simulations and parametric analysis. This step was performed simultaneous to the experiment testing to reduce the time effort for the procedure.

\subsection{Design of Experiments}

Design of Experiments was very advanced and efficient methodology to find the prominent factors, component selection and variability analysis of the component. The prominent approach, Taguchi method was implemented here. In order to get best out of design of experiments, a modified methodology was designed as two-step DOE. In general, there was uncertainty in selecting the stress factors for design of experiments and accelerated testing. Hence, at first screening step, the test was designed to know variability of stress on the effect of performance parameters. In the second testing step, the levels of the stress were aggressive which defines the degradation of the performance parameters.

\subsection{Accelerated Testing}

The input pattern obtained for degradation from modified design of experiments was applied in the accelerated testing step [13]. As from the analysis, this particular pattern leads to further degradation over the accelerated time.

\subsection{Regressions and Failure Modeling}

The data collected from both design of experiments and accelerated testing was used for statistical data and modeling analysis using various methods such as response surface regressions, regression methods, support vector regression and other tools to quantify the stress parameters and its behavior on the performance of the device. This data was also useful for failure models obtained from failure mechanisms.

\subsection{Compare prediction models}

The reliability indices calculated from traditional approaches, physics of failure models, models generated from statistical analysis and simulations were compared in this step. In accordingly, the reliability growth techniques were implemented and appropriately the repair/replacement mechanism was discussed. The actual decision was selected on basis of available inventory, importance of the component, resources available, selection of vendor and several other factors such as risk, uncertainty and human factors.

\subsection{Factor Calculations}

In parallel, the factors like time taken, human factors and risk was calculated at each step. With regarding the cost, this cost only provides the prediction cost of the entire block diagram in Fig 2. The total cost thus calculated including the penalty cost that was incurred by the repair/replacement costs. In the most of electronic components, the probability of replacement was more than the repair. In this case study, we are considering the replacement cost of the component.

\section{FAILURE STUDIES OF COMPONENTS}

\subsection{Instrumentation Amplifier}

An instrumentation amplifier is a gain differential device that provides the output with high common mode rejection ratio and high accuracy. This differential amplifier is widely used in several electronic applications even precise environments and also considered as most versatile amplifier. This device (INA118) require very high input impedance, low bias and offset currents, low noise and balanced inputs in order to minimize common mode gain. The basic building blocks of the instrumentation amplifier are op-amps. Technically, it is equivalent to standard op-amp, the each of the inputs of it is driven by two another op-amps to buffer the inputs and to produce desired output for impedance matching.

The gain of the amplifier is defined as the output voltage divided by input voltage [10]

$$
G=\text { Vout } /(V 1-V 2)=(1+2 R 1 / \text { Rgain }) R 3 / R 2
$$

Since, the voltage across Rgain equals Vin (V1-V2), the current through Rgain will equal (VIn/Rgain). Amplifiers A1 and A2 on left side will operate with gain and amplify the input signal. Rgain can be useful to tune for desired gain without effecting common mode gain and error. The 


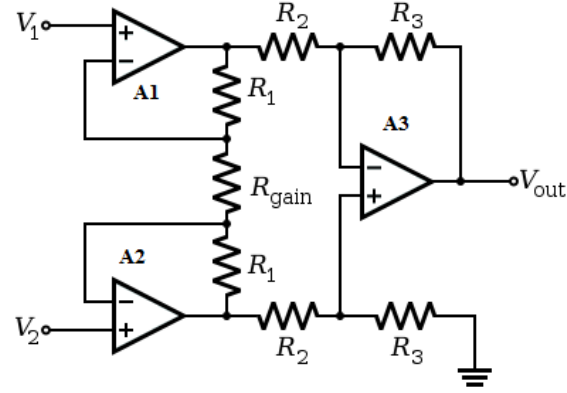

Fig 3: Test circuit for Instrumentation Amplifier, INA118

differential signal will be increased by gain, but the commonmode error will not increases, so the ratio (Gain (VDIFF)/(VError CM)) will increase. Thus, CMRR will theoretically increase in direct proportion to gain - a very useful property. CMMR is the tendency of the devices to reject the input signals common to both input leads. Ideally, a differential amplifier takes the voltages V1 and V2 on its two inputs and produces an output voltage

$$
\text { Vout }=A d(V 1-V 2)
$$

where Ad is the differential gain. But the output of a real differential amplifier is described as

$$
\text { Vout }=A d(V 1-V 2)+\frac{1}{n} A c m(V 1+V 2)
$$

where Acm is the common-mode gain, which is typically much smaller than the differential gain. Thus CMRR is defined as the ratio of the powers of the differential gain over the common-mode gain, measured in decibels

$$
C M M R=10 \log _{10}\left(\frac{A d}{A c m}\right)^{2}=20 \log _{10}\left(\frac{A d}{|A c m|}\right)
$$

\subsubsection{Effect of Input Voltage Difference}

The both gain and CMMR equations were considered as performance parameters and sensitive to the input voltage difference that further degrades the amplifier. Hence, the input voltage was selected as stress parameter.

\subsubsection{Effect of Temperature}

The temperature dependence of bipolar transistors depends on a multitude of parameters affecting the bipolar transistor characteristics in different ways. Important effect is the temperature dependence of the current gain. Since the current gain depends on both the emitter efficiency and base transport factor [15].

The emitter efficiency depends on the ratio of the carrier density, diffusion constant and width of the emitter and base. As a result, it is not expected to be very temperature dependent. The carrier densities are linked to the doping densities. Barring incomplete ionization, which can be very temperature dependent, the carrier densities are independent of temperature as long as the intrinsic carrier density does not exceed the doping density in either region. The width is very unlikely to be temperature dependent and therefore also the ratio of the emitter and base width. The ratio of the mobility is expected to be somewhat temperature dependent due to the different temperature dependence of the mobility in n-type and p-type material.

Leakage current ICO and $\beta$ increase with temperature. The DC $\beta$ hFE increases exponentially. The AC $\beta$ hfe increases, but not as rapidly. It doubles over the range of $-55^{\circ}$ to $85^{\circ} \mathrm{C}$. As temperature increases, the increase in $h f e$ will yield a larger common-emitter output, which could be clipped in extreme cases. The increase in $h F E$ shifts the bias point, possibly clipping one peak. The shift in bias point is amplified in multi-stage direct-coupled amplifiers. The solution is some form of negative feedback to stabilize the bias point. This also stabilizes AC gain [11].

As from the studies from BJT technology, temperature and radiation is selected as stress parameters. The emitter and collector current of npn BJT is given as Equation (5) and (6).

$$
\begin{gathered}
I_{E}=I_{E S}\left(e^{\frac{V B E}{V T}}-1\right) \\
I_{C}=\alpha_{T} I_{E S}\left(e^{\frac{V B E}{V T}}-1\right)
\end{gathered}
$$

The output voltage VCE is given as in Equation

$$
V_{C E}=V_{C E}-I_{C} R_{e f f}
$$

Where $\mathrm{R}_{\text {eff }}$ is effective output resistance at the output, $\mathrm{I}_{\mathrm{ES}}$ $=$ reverse saturation current at base-emitter diode, $\alpha_{\mathrm{T}}=$ common base forward short circuit gain, $\mathrm{V}_{\mathrm{T}}=$ Thermal Voltage $\mathrm{kT} / \mathrm{q}, \mathrm{V}_{\mathrm{BE}}=$ base-emitter Voltage, $\mathrm{V}_{\mathrm{CE}}=$ basecollector Voltage, $\mathrm{V}_{\mathrm{CC}}=$ Source Voltage typically $5 \mathrm{~V} / 10 \mathrm{~V}$.

In Eber-Moll Model, $I_{C}$ grows at about $9 \% /{ }^{0} \mathrm{C}$ if you hold $V B E$ constant and $V B E$ decreases by $2.1 \mathrm{mV} /{ }^{\circ} \mathrm{C}$ if you hold IC constant with the temperature.

Since both the currents depend on temperature parameter $V_{T}$, the raise in the temperature leads to vary these parameters which finally lead to degrade the performance of op-amps in turn the instrumentation amplifier. Hence, temperature was considered as another stress parameter which leads to reduce the gain and CMMR of instrumentation amplifier. In this paper, we selected IN128 for failure investigation. Since there was no possible failure mechanism associated with it in the literature, the failure model was not considered. But from this root cause analysis, the appropriate information was drawn out to make further testing.

The failure mechanism involved in the instrumentation was the degradation of the device parameters like gains, thermal voltage and intrinsic voltage and currents. This failure could be assessed using the standard failure analysis method which is electrical characterization.

\subsection{BJT Transistor}

In this study we selected 2N2222, a normal BJT transistor for failure study. At the field, this component was exposed to nuclear radiation and hence we interested to test the IC for radiation. As BJT technology was sensitive to the temperature as described in case of instrumentation amplifier, we are considering it as another stress parameter. Even in this case also, there were no failure models for failure mechanisms observed from the literature, the methodology was continued on the information gathered from the root cause analysis and field environment failed data. For further hypothesis testing, experimentation was conducted to achieve the results of 
failure data. The test circuit of 2N2222 is given below in Fig 4. The stress parameters of BJT transistor was selected as temperature and radiation.

\subsubsection{Effect of Radiation}

Another stress parameter which degrades the BJT devices is $\beta$-radiation. Degradation of many types of bipolar transistors and circuits is known to depend strongly on dose rate. For a given total dose, degradation is more severe in low dose rate exposure than high dose rate exposure [12]. This effect has been attributed to space charge effects from trapped holes and hydrogen related species through oxygen vacancies in base oxide. There are several hardness assurance tests and most popular has been high dose rate irradiation at elevated temperatures.

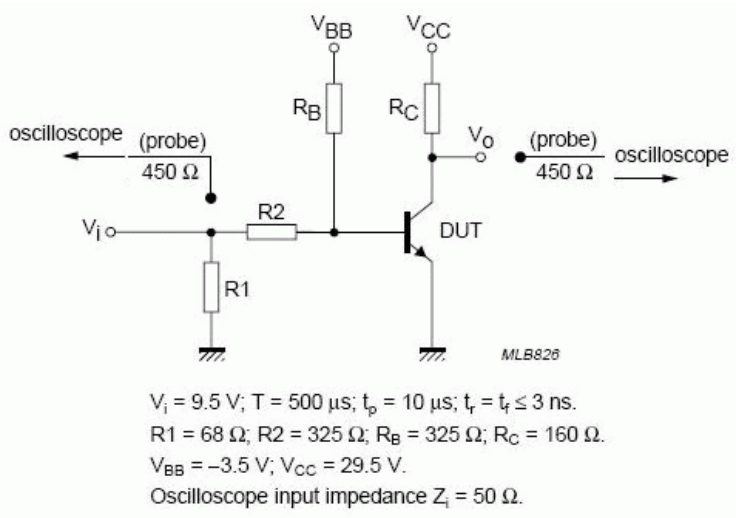

Figure 4: Test circuit for 2N2222 BJT transistor

\section{RELIABILITY PREDICTIONS}

By applying the above modified methodology for reliability prediction, reliability indices of both the devices were calculated using MILHDBK 217+ and by using experimental analysis considering the accelerated testing since there were no standard physics of failure models available for developing failure model. The work also carried out the simulation results to verify the behavior of both the stress parameters on the subject of interested component.

\subsection{Instrumentation Amplifier}

Using RIAC $217+$ [7], the failure rate equation for plastic encapsulated integrated circuit was:

$$
\begin{gathered}
\lambda_{P}=\pi_{G}\left(\lambda_{O B} \pi_{D C O} \pi_{T O}+\lambda_{E B} \pi_{D C N} \pi_{R H T}+\lambda_{T C B} \pi_{C R} \pi_{D T}\right)+ \\
\lambda_{S I B} \pi_{S I D T}+\lambda_{E O S}
\end{gathered}
$$

Where $\lambda_{\mathrm{P}}=$ Predicted failure rate, $\pi_{\mathrm{G}}=$ Reliability growth rate multiplier, $\lambda_{\mathrm{OB}}=$ base failure rate, $\pi_{\mathrm{DCO}}=$ failure rate multiplier of duty cycle, $\pi_{\mathrm{TO}}=$ failure rate multiplier for temperature, $\lambda_{\mathrm{EB}}=$ environmental base failure rate, $\pi_{\mathrm{DCN}}=$ failure rate multiplier of duty cycle for non operating, $\pi_{\mathrm{RHT}}=$ failure rate multiplier for temperature-humidity, $\lambda_{\mathrm{TCB}}=$ base failure rate for temperature cycling, $\pi_{\mathrm{DCO}}=$ failure rate multiplier for cycling rate, $\pi_{\mathrm{DT}}=$ failure rate multiplier for delta temperature, $\lambda_{\mathrm{SIB}}=$ base failure rate for solder joint,,$\pi_{\mathrm{DT}}$ $=$ failure rate multiplier for solder joint delta temperature and $\lambda_{\mathrm{EOS}}=$ failure rate overstress.
There is no specific assigned model for the instrumentation amplifier. Based on its internal diagram, there are 2 portions for analysis to be needed: precision amplify and overload-protection. Comparatively, overload protection was smaller than the precision amplifier and hence precision amplifier was only analyzed. Selecting the required parameters for the INA118 instrumentation amplifier, the MTTF was calculated as $67 \times 10^{6} \mathrm{hrs}$.

The test circuit for instrumentation amplifier in Fig 3 was subjected to both temperature and voltage simultaneously using set of runs depicted by design of experiments. From the experimentation, the stress levels of temperature $55^{\circ} \mathrm{C}$ and voltage $11 \mathrm{~V}$ produces higher degradation than other set of stress levels. These values were considered as input to the accelerated testing for extended period of time. The output voltage in terms of temperature, input stressed voltage and accelerated time was generated by response surface regression and calculated by using equation (9)

$$
\begin{aligned}
& \text { Vout }=-4.4588+0.00029 T-4.035 t+0.033+ \\
& 5.546 \times 10^{-6} T^{2}+3.797 \times 10^{-6} t^{2}-6.624 \times 10^{-4} V^{2}+ \\
& 4.459 \times 10^{-5} T V+0.00012 t T
\end{aligned}
$$

By considering the $5 \%$ degradation as failure in the gain and normal operating temperatures of temperature, the MTTF was calculated as $52 \times 10^{7}$ hrs. There was reduction in MTTF figure when compared to traditional prediction because this experimentation considers another stress parameter as input voltage which reduces the degradation of the output variable. The CMMR of the instrumentation was also degraded when applied to the stress parameters within 5-10\% with respective stress levels. The simulation studies verified the behavior of both stress parameters on degradation of output variables.

\subsection{BJT Transistor}

Using RIAC 217+ [7], the failure rate equation for transistors was:

$$
\begin{gathered}
\lambda_{P}=\pi_{G}\left(\lambda_{O B} \pi_{D C O} \pi_{T O} \pi_{S}+\lambda_{E B} \pi_{D C N} \pi_{T E}+\lambda_{T C B} \pi_{C R} \pi_{D T}\right)+ \\
\lambda_{S I B} \pi_{S I D T}+\lambda_{I N D}
\end{gathered}
$$

Where, $\pi_{\mathrm{S}}=$ stress failure rate multiplier, $\pi_{\mathrm{TE}}=$ failure rate multiplier, temperature environment and $\lambda_{\mathrm{IND}}=$ induced failure rate. The remaining factor representation was same as in Eqn .By selecting and calculating the above equation, the MTTF was calculated as $1.5 \times 10^{6} \mathrm{hrs}$.

The test circuit for BJT transistor was subjected to temperature and then radiation as these parameters cannot be applied simultaneously. The run for stress levels for maximum degradation were temperature as $90^{\circ} \mathrm{C}$ and radiation exposure of $1 \mathrm{M} \mathrm{rad}$. The voltage was generated by using response surface regression and shown below in equation

$$
\begin{aligned}
& \text { Vout }=-2.321+0.00027 T-3.035 t+0.0452 R+ \\
& 5.235 \times 10^{-6} T^{2}+2.535 \times 10^{-6} t^{2}-5.644 \times 10^{-4} R^{2}+ \\
& 4.487 \times 10^{-5} T R+0.00026 t R
\end{aligned}
$$

Under the normal conditions with $5 \%$ degradation as failure, MTTF was calculated as $1.2 \times 10^{7} \mathrm{hrs}$. The gain over the traditional methodology was that the physics of failure considers the actual stress parameter in this case, the radiation. 


\subsection{Life Costing Calculations}

For the instrumentation amplifier, the initial costs of both prediction methodologies were produced (in Indian equivalent of dollars). The initial cost of traditional methodology was

$$
C_{i t}=C_{h d b k \mid e q v}
$$

Where $\mathrm{C}_{\text {it }}=$ initial cost for traditional methodology and $\mathrm{C}_{\text {hdbkleqv }}=$ cost of handbook for equivalent component. The actual cost of RIAC 217 plus handbook was 200\$. The assumption was that the figure of each calculation was 200 . Hence, $C_{i t}$ was assumed as $1 \$$. The cost of physics of failure methodology was calculated as

$$
C_{i p}=C_{e r}+C_{r s}+C_{f a}+C_{e x p}+C_{\text {model }}
$$

Where $C_{\text {ip }}=$ initial cost for physics of failure methodology, $\mathrm{C}_{\mathrm{er}}=$ expert reviews as consultation fee, $\mathrm{C}_{\mathrm{rs}}=$ cost of research papers, $\mathrm{C}_{\mathrm{fa}}=$ cost of failure analysis, $\mathrm{C}_{\mathrm{exp}}=$ cost of experimentation includes design, fabrication of $\mathrm{PCB}$ boards, sources and measurement instruments and other miscellaneous and $\mathrm{C}_{\text {model }}=$ cost of tools required for modeling. By considering the figure of each calculation as 200 , the total cost consumed by physics of failure methodology was $\mathrm{C}_{\mathrm{ip}}=$ $50+120+50+250+30=500 \$$ (approximately).

Similarly, the initial or prediction costs of BJT transistor was calculated as $\mathrm{C}_{\mathrm{it}}=1 \$$ and $\mathrm{C}_{\mathrm{ip}}=500 \$$.

For calculating the penalty costs, it was needed to consider costs of the instrumentation amplifier as $\mathrm{C}_{\mathrm{IA}}=15 \$$ and cost of BJT transistor as $C_{\mathrm{BT}}=1 \$$, time period and also the number of replacements over time. Hence, penalty costs were computed for both the components. For instrumentation amplifier, the cost of penalty cost by using traditional method,

$$
C_{p t}=C c\left(1+\frac{(1+i)^{t}-1}{i(1+i)^{t}} * 2 * \frac{t 0}{M T T F_{t}}\right)
$$

Where $\mathrm{C}_{\mathrm{c}}=$ cost of the component, MTTFt $=$ time to failure using traditional methodology, $\mathrm{Cf}=2 * \mathrm{Cc}=$ failure cost as it was assumed as doubled for traditional methods, $\mathrm{i}=$ interest rate, $\mathrm{t} 0=$ age of replacement $=1$ (assumed) and $\mathrm{t}$ is the design life. Similarly the cost of penalty using physics of failure methodology was shown in equation.

$$
C_{p p}=C c\left(1+\frac{(1+i)^{t}-1}{i(1+i)^{t}} * \frac{t 0}{M T T F_{p}}\right)
$$

Where MTTFp = time to failure by physics of failure.

The total cost of the reliability prediction was summation of initial and penalty costs. For traditional prediction methodology,

$$
C_{t t}=C_{i t}+C_{p t}
$$

Similarly for physics of failure prediction, the total cost was

$$
C_{p p}=C_{i p}+C_{p p}
$$

To compare the total cost incurred by both the prediction methods for both the components, it was assumed that the design life was 10 years and ratio of number of replacements for a component was equal to the ratio of individual time to failures.

$$
\frac{n_{p}}{n_{t}}=\frac{M T T F_{p}}{M T T F_{t}}
$$

By considering these assumptions, for an instrumentation amplifier, the total cost expended on traditional methodology (1052) was more than the proposed methodology (650) for $n$ replacements. But for the BJT transistor, the total cost expended on traditional methodology (88) was less than the proposed methodology (508) for $\mathrm{n}$ replacements.

\subsection{Other Factors}

In the traditional reliability prediction, there was a certain amount of risk associated with it and uncertainty over the selection of several standard books available. There was no possible inclusion of human factors, needed less amount of time, space and man power. But in the case of physics of failure reliability prediction methodology, it requires large amount of time, space, man power and human factors regarding the implementation of experimentation and modeling. Also, it provides the approximate figure of the failure time and had higher accuracy than previous prediction models. The difference between both the prediction methods was tabulated as in table [2].

\section{Table 2: Comparison of Reliability Prediction methods}

\begin{tabular}{|l|l|l|}
\hline Factor & Traditional & PoF \\
\hline Initial Cost & Low & High \\
\hline Penalty Cost & High & Low \\
\hline Total Cost for Critical & High & Low \\
\hline Total Cost for non-critical & Low & High \\
\hline Time & Less & Large \\
\hline Space & Less & Large \\
\hline Expertise & Low & High \\
\hline Human factors & Less & More \\
\hline Risk & High & Low \\
\hline Uncertainty & High & Low \\
\hline Man Power & Few & Large \\
\hline
\end{tabular}

\section{REFERENCES}

1. "European power supply manufacturers association, MTBF Report", June 2005

2. "Military Handbook, Reliability prediction of electronic component, MIL-HDBK-217F"

3. Jeff Jones, Joseph Hayes, "A Comparison of ElectronicReliability Prediction Models", IEEE Transactions on Reliability, vol. 48, no. 2,1999 june

4. B. Foucher, J. Boullie, B. Meslet, D. Das, "A review of reliability prediction methods for electronic devices", Microelectronics Reliability 42 (2002) 1155-1162

5. "Failure Mechanisms and Models for Semiconductor Devices", JEDEC Publication, JEP122E, Originally published as JEP122D.01 March 2009

6. "Semiconductor Device Reliability Failure Models", International SEMATECH, May 31, 2000

7. "Handbook Of 217Plus Reliability Prediction Models, Reliability Information Analysis Center (RIAC)", 26 May 2006

8. Lloyd W. Condra, "Reliability improvement with design of experiments", 2nd edn, Marcel Dekker, 2001. 
9. Perry L Martin, "Electronic Failure Analysis Handbook, Techniques and Applications for Electronic and Electrical Packages, Components, and Assemblies", McGraw Hill, 1999

10. Charles Kitchin, Lew Counts, “A Designer's guide to Instrumentation Amplifiers", Analog Devices, 2006

11. Guijie Wang, Gerard C.M. Meijer, "The temperature characteristics of bipolar transistors fabricated in CMOS technology", Elsevier, Sensors and Actuators 872000 8189.

12. S.C. Witczak, R.D. Schrimpf, D.M. Fleetwood, K. F. Galloway, R.C.Lacoe, D.C. Mayer, J.M. Puhl, R.L.Pease, J.S. Suehle, "Hardness assurance testing of bipolar junction transistors at elevated irradiation temperatures", IEEE transaction on Nuclear Science, Vol 44, No. 6, Dec 1997

\section{BIOGRAPHIES}

Adithya Thaduri: He studied electronics and instrumentation engineering in bachelors and reliability engineering in masters. Presently, he is doing PhD at IIT Bombay India and Lulea University of Technology, Sweden in association with Bhabha Atomic Research center India. His core area of research on predicting the life of critical electronic components using physics of failure approach.

A.K. Verma received the B.Tech (Hons) and Ph.D. (Engg.) degrees from Department of Electrical Engineering, IIT Kharagpur. He has been with IIT Bombay as a faculty since
1988. He is a professor in the Department of Electrical Engineering at IIT Bombay. He has over 180 research papers to his credit and has supervised twenty eight $\mathrm{Ph} . \mathrm{D}$. thesis and eight four Masters Thesis at IIT Bombay. He is the Editor in Chief of the International Journal of Systems Assurance and Engineering Management. He has been a guest editor of special issues of various international journals $\mathrm{He}$ is a senior member of IEEE and life fellow of IETE. His research interests on reliability engineering include interdisciplinary applications in software engineering, computing, maintenance, and power systems.

Uday Kumar obtained his B. Tech from India during the year 1979. After working for 6 years in Indian mining industries, he joined the postgraduate program of Lulea University of Technology, Lulea, Sweden and obtained a $\mathrm{PhD}$ degree in field of Reliability and Maintenance during 1990. He worked as a Senior Lecturer and Associate Professor at Lulea University 1990-1996. In 1997, he was appointed as a Professor of Mechanical Engineering (Maintenance) at University of Stavanger, Stavanger, Norway. Since July 2001, he has taken up the position as a Professor of Operation and Maintenance Engineering at Lulea University of Technology, Lulea, Sweden. Currently he is the Director of Lulea Railway Research Center. His research interests are equipment maintenance, reliability and maintainability analysis, etc. He is also member of the editorial boards and reviewer for many international journals. He has published more than 150 papers in International Journals and Conference Proceedings. 